De Reuck J. Int J Geriatr Gerontol 5: 127.

www.doi.org/10.29011/2577-0748.100027

www.gavinpublishers.com

\title{
Multiple System Atrophy: An Update Review
}

\section{Jacques De Reuck*}

Department of Neurology, University Hospital, Ghent, Belgium

"Corresponding author: Jacques De Reuck, Department of Neurology, University Hospital, Ryvissche park 16, 9052 Zwijnaarde, Belgium.

Citation: De Reuck J (2021) Multiple System Atrophy: An Update Review. Int J Geriatr Gerontol 5: 127. DOI: 10.29011/25770748.100027

Received Date: 18 December, 2021; Accepted Date: 27 December, 2021; Published Date: 31 December, 2021

\begin{abstract}
Multiple system atrophy is a progressive fatal neurodegenerative disease, characterized alpha-sunucleine accumulation mainly in oligodendroglial cells of the white matter and to a lesser degree in neurons. Autonomic and urogenital failures are the dominant clinical features. The disease is subdivided in a predominant parkinsonian type and in a predominant cerebellar atactic type. The parkinsonian type represents $80 \%$ of the patients while the cerebellar type is found in $20 \%$ of the cases. Smoking is less frequent in patients with the parkinsonian type of multiple system atrophy. On magnetic resonance imaging middle cerebellar peduncle atrophy and hyper intensity, putaminal hypointensity and hyperintense putaminal rim in addition to pons hot bun sign and atrophy are the main features. No protective treatment is available. Only supportive measures can be proposed.
\end{abstract}

Keywords: Multiple system atrophy; Alpha-synucleine; Parkinsonian type; Cerebellar atactic type; Parkinson's disease; Dementia with Lewy bodies

Abbreviations: MSA: Multiple System Atrophy; MCA-P: Parkinsonian type of Multiple System Atrophy; MCA-C: Cerebellar type of Multiple System Atrophy; PD: Parkinson's disease

\section{Introduction}

The new criteria retain the diagnostic categories of Multiple System Atrophy (MSA) with predominant Parkinsonism features (MSA-P) and MSA with predominant cerebellar ataxia (MSA-C) to designate the predominant motor features and also the designations of definite, probable, and possible MSA [1].

Alpha-synuclein is observed in oligodendroglial cytoplasmic inclusions of the cerebral white matter in MSA brains. This protein may be responsible to form filaments that aggregate into cytoplasmic inclusions, contributing to the dysfunction or death of glial cells as well as neurons in the different MSA phenotypes [2]. Using cryo-electron microscopy, two different types of alphasynuclein inclusions are observed, each of which consists of two different protofilaments [3].

Synucleinoparthies include not only multiple system atrophy but also Parkinson's Disease (PD) and dementia with
Lewy bodies due to the presence of alpha-synuclein aggregates throughout the nervous system. However, they diverge from one another with regards to their clinical and pathological phenotype. Although there are many similarities of the alpha-synucleair pathology between MSA and PD, the former is significantly more potent in inducing motor deficits and degeneration of the striatum. The more widened spreading of the alpha-nuclear pathology and inflammation reflects the more aggressive nature of MSA [4]. Perturbed iron homeostasis is a potential factor in the pathogenesis in MSA-associated neurodegeneration [5].

A controversial topic in MSA is the non-supporting feature of the association of cognitive impairment from both clinical and pathological perspectives $[6,7]$.

\section{Multiple System Atrophy with Predominant Parkinsonian Features}

The interval from initial symptom to combined motor and autonomic dysfunction predicts the functional deterioration and survival in MSA patients. MSA-P patients have more rapid functional deterioration than the MSA-C ones. Isolated autonomic failure and REM sleep behavior disorder are common premotor features of MSA [8]. MSA is dominated by autonomic and urogenital failure. The MSA-P type represents $80 \%$ [9].

On Magnetic Resonance Imaging (MRI) the hyperintense rim at the lateral edge of the dorsolateral putamen is observed in 
$34.5 \%$ and the hot cross bun sign in the pontine basis in $63.3 \%$ [7]. Putaminal iron accumulation is a secondary by-product of the neurodegenerative atrophy [10].

Patients with MSA-P show normal neuromelanin in the substatia nigra and the locus coeruleus, similar to normal brains and allowing the distinction compared to idiopathic Parkinson ones [11]. The pontine hot-cross bun is of lower visual grade in MSA-P than in MSA-C on T2*weighted imaging [12].

The incidence of smokers is significantly lower in the MSA-P patients than in other non-Parkinson related diseases [13]. Rare MSA-P cases can be associated to adult polyglucosan body disease [14].

\section{Multiple system atrophy with predominant cerebellar features.}

The MSA-C represents approximately $20 \%$ of the whole disease group [9]. Middle cerebellar peduncle atrophy and hyperintensity, hot bun sign, putaminal hypointensity and hyperintense putaminal rim in addition to pons atrophy are the main features [15].

The alpha-synucleinopathy of the oligodendroglial cells involves mainly the olivopontocerebellar and the autonomic nervous systems, but also to a lesser degree other parts of the central and peripheral nervous systems [16,17].

DAT-scan imaging with loflupane123 injection, however, reveals bilateral nigro-striatal degeneration in possible MSA-C, due to a decreased uptake of dopamine transporters [18].

\section{Therapeutical Options}

There are many drug trials in MSA. Most studies include those for relieve of parkinsonian motor symptoms, autonomic dysfunction, and depression [19].

Only one third of MSA patients may benefit from dopamine for symptomatic treatment of parkinsonism, whereas physiotherapy remains the best therapeutic option for cerebellar ataxia. Many curative treatment trials are available for MSA [20]. In a small series of MSA patients, receiving amantadine $200 \mathrm{mg}$ twice daily, there is a trend of reduction of the parkinsonian symptoms [21].

A preliminary study shows that intravenous amantadine is a safe and effective treatment in MSA-P. However still an additional double-blind placebo-controlled study is needed to confirm these results [22].

Riluzole is generally well tolerated but there are no significant anti-parkinsonian effects. Treatment with $1 \mathrm{mg}$ riluzole daily do not improve the population of patients with MSA-P [23]. A treatment with $1 \mathrm{mg}$ rasagiline daily does not improve the population of patients with MSA-P [24].

In a mouse model of MSA rifampicin inhibits the formation alfa-synuclien fibrils. That is why a double-blind study in MSA patients was started at a dose of two times $300 \mathrm{mg}$ daily. Rifampicin did not slow or halt the progression of the disease [25].
Polyphenol epigallocatechin gallate inhibits alpha-synuclein aggregation and reduces associated toxicity. However, 48 weeks of treatment do not modify disease progression in patients with MSA. Epigallocatechin gallate is overall well tolerated but hepatotoxic effects are observed in some patients [26].

\section{Conclusions}

Multiple system atrophy is a fatal progressive neurodegenerative disease for which no conclusive protective treatment is available. Only supporting measures can be proposed.

\section{References}

1. Gilman S, Wenning GK, Low PA, Brooks DJ, Mathias CJ, et al. (2008) Second consensus statement on the diagnosis of multiple system atrophy. Neurology 71: 670-676.

2. Tu PH, Galvin JE, Baba M, Giasson B, Tomita T, et al. (1998) Glial cytoplasmic inclusions in the white matter oligodendrocytes of multiple system atrophy brains contain insoluble alpha-synuclein. Ann Neurol 44: 415-422.

3. Schweighauser M, Shi Y, Tarutani A, Kametami F, Murzin AG, et al. (2020) Structures of alpha-synuclein filaments from multiple system atrophy. Nature 585: 464-469.

4. Van der Perren A, Gelders G, Fenyi A, Boosset L, Brito F, et al. (2020) The structural differences between patient-derived alpha-synuclein strains dictate characteristics of Parkinson's disease, multisystem atrophy and dementia with Lewy bodies. Acta Neuropathol 139: 9771000.

5. Kaindlstorfer C, Jellinger KA, Eschlblock S, Stefanova N, Weiss G, et al. (2018) The relevance of iron in the pathogenesis of multiple system atrophy: a viewpoint. J Alzheimers Dis 61: 1253-1273.

6. Koga S, Dickson DW (2018) Recent advances in neuropathology, biomarkers and therapeutic approach of multiple system atrophy. J Neurol Neurosurg Psychiatry 89: 175-184.

7. Fanciulli A, Stankovic I, Krismer F, Seppi K, Levin J, et al. (2019) Multiple system atrophy. Rev Neurobiol 149: 137-192.

8. Wenning GW, Geser F, Stampfer-Kountchev M, Tison F (2003) Multi system atrophy: an update. Mov Disord 18: S34-S42.

9. Watanabe H, Saito Y, Terao S, Ando T, Kachi T, et al. (2002) Progression and prognosis in multiple system atrophy: an analysis of 230 Japanese patients. Brain 125: 1070-1083.

10. Lee MJ, Kim T-H, Kim SJ, Mun C-W, Shin J-H, et al. (2019) Speculating the timing of iron deposition in the putamen in multisystem atrophy. Parkinsonism Relat Disord 63: 106-110.

11. Simoes RM, Caldas AC, Grilo J, Correia D, Guerreiro C, et al. (2020) A distinct neuromelanin magnetic resonance imaging pattern in parkinsonian multiple system atrophy. BMC Neurol 20: 432.

12. Deguchi K, Ikeda K, Kume K, Takata T, Kokudo Y, et al. (2015) Significance of the hot-cross bun sign on T2*-weighted MRI for the diagnosis of multiple system atrophy. J Neurol 262: 1433-1439.

13. Tseng F-S, Deng K, Ong Y-L, Li H-H, Tan E-K (2020) Multiple System Atrophy (MSA) and smoking: a meta-analysis and mechanistic insights. Aging (Albany NY) 12: 21959-21970. 
14. Krim E, Vital A, Macia F, Yekhlef F, Tison F (2005) Atypical parkinsonism combining alpha-synuclein inclusions and polyglucosan body disease. Mov Disord 20: 200-204.

15. Carré G, Dietermann JL, Gebus O, Montaut S, Lagha-Boukhiza O, et al. Brain MRI and multiple system atrophy of cerebellar type: a prospective study with implications for clinical diagnosis. J Neurol 267: 1269-1277.

16. Ahmed Z, Asi YT, Sailer A, Lees AJ, Houlden H, et al. (2012) The neuropathology, pathophysiology and genetics of multiple system atrophy. Neuropathol Appl Neurobiol 38: 4-24.

17. Jellinger KA (2018) Multiple system atrophy: an oligodendroglioneural synucleinopathy 1. J Alzheimers Dis 62: 1141-1179.

18. Bellini G, Del Prete E, Unti E, Frosini D, Siciliano G, et al. (2021) Positive DAT-scan in SPG7: a case report mimicking possible MSA-C. BMC Neurol 21: 328.

19. Rey MV, Perez-Lloret S, Pavy-Le Traon A, Meissner WG, Tison F, et al. (2014) A cross-sectional study on drug use in multiple system atrophy. CNS Drugs 28: 483-490.

20. Perez-Lloret S, Flabeau O, Fernagut P-O, Pavy-Le Traon A, Rey MV, et al. (2015) Current concepts in the treatment of multiple system atrophy. Mov Disord Clin Pract 2: 6-16.
21. Wenning GK, Working Group on Atypical Parkinsonism of the Austrian Parkinson's Society (2005) Placebo-controlled trial of amantadine in multi-system atrophy. Clin Neuropharmacol 28: 225-227.

22. Friedberg A, Erikh H, Nassar M, Sprecher E, Schlesinger I (2018) Efficacy of parenteral amantadine therapy in the treatment of multiple system atrophy with predominant parkinsonism. Clin Neuropharmacol 11: $160-163$.

23. Seppi K, Peralta C, Diem-Zangeri A, Puschban Z, Mueller J, et al. (2006) Placebo-controlled trial of riluzole in multiple system atrophy. Eur J Neurol 13: 1146-1148.

24. Poewe W, Seppi K, Fitzer-Atras CJ, Wenning GK, Gilman S, et al. (2015) Efficacy of rasagiline in patients with the parkinsonian variant of multiple system atrophy: a randomised, placebo-controlled trial. Lancet Neurol 14: 145-151.

25. Low PA, Robertson D, Gilman S, Kaufmann H, Singer W, et al. (2014) Efficacy and safety of rifampicin for multiple system atrophy: a randomised, double-blind, placebo-controlled trial. Lancet Neurol 13: 268275.

26. Levin J, Maaß S, Schubert M, Giese A, Oertel WH, et al. (2019) Safety and efficacy of epigallocatechin gallate in multiple system atrophy (PROMESA): a randomised, double-blind, placebo controlled trial. Lancet Neurol 18: 724-735. 